\title{
Evaluation of Solanum sisymbriifolium as a Potential Inoculum Source of Verticillium dahliae and Colletotrichum coccodes
}

Z. A. Frederick and T. F. Cummings, Department of Plant Pathology, Washington State University, Pullman, 99164; C. R. Brown and R. A. Quick, United States Department of Agriculture, Agricultural Research Service, Prosser, WA 99350; and D. A. Johnson, Department of Plant Pathology, Washington State University, Pullman, 99164

\begin{abstract}
Solanum sisymbriifolium, the litchi tomato, is a perennial herbaceous plant from South America that is used as a trap crop to reduce soilborne populations of the pale cyst nematode Globodera pallida, an important potato pathogen. Possible interactions of soilborne potato pathogens Verticillium dahliae and Colletotrichum coccodes with litchi tomato are unknown, yet important for potato production if litchi tomato is to be planted as a trap crop. The goal of this research was to quantitatively assess if litchi tomato is a potential inoculum source for $C$. coccodes and $V$. dahliae by comparing colony forming units (CFU) observed in litchi tomato to susceptible and resistant potato cultivars. The potato cvs. Alturas $(P=0.0003)$, Ranger Russet $(P=0.0193)$, and Russet Norkotah

$(P=0.0022)$ produced more CFUs of the potato pathotype of $V$. dahliae than litchi tomato the first of two years of greenhouse trials. Significantly more CFUs of the potato pathotype of $V$. dahliae were quantified from stems and roots of only cv. Russet Norkotah compared with litchi tomato $(P=0.0001)$ in the second year. The CFUs for $C$. coccodes varied between litchi tomato and the potato cvs., perhaps due to varying levels of resistance since litchi tomato is from a selected intermated seed source. Based on these data, the effect of litchi tomato in rotation with potato is likely to have limited effect on the proliferation of $V$. dahliae or $C$. coccodes populations in the soil when compared with a susceptible potato cultivar.
\end{abstract}

Two important diseases of potato in the northwestern United States are Verticillium wilt and black dot, caused by the soilborne fungi Verticillium dahliae Kleb. and Colletotrichum coccodes Wallr., respectively. V. dahliae infects a wide range of plants, making it one of the most important pathogens of dicotyledonous crop plants in North America (Agrios 2005; Bhat and Subbarao 1999; Dung et al. 2013). Despite the wide host range of $V$. dahliae, individual isolates vary in aggressiveness when introduced to different plant hosts under controlled conditions, which has also been noted from field isolations (Bhat and Subbarao 1999; Dung et al. 2013; Pegg and Brady 2002). Host adaptation of $V$. dahliae may occur over a relatively short period of time within both annual and perennial crop hosts such as pepper and mint, respectively (Bhat et al. 2003; Fordyce and Green 1960). Individual $V$. dahliae isolates that are aggressive to a particular host have been called host-adapted pathotypes (Dung et al. 2013; Pegg and Brady 2002). Certain $V$. dahliae isolates that are grouped into host-adapted pathotypes often fall into certain vegetative compatibility groups (VCGs; Katan 2000), although this is not true for all isolates (Bhat and Subbarao 1999; Dung et al. 2013). The VCGs of $V$. dahliae are scored by complementation of nitrate-nonutilizing (nit) mutants that are generated by culture on $1 \%$ potassium chlorateamended medium and able to fuse and grow on media with defined nitrogen sources (Joaquim and Rowe 1990, 1991).

The complete host range of another important soilborne plant pathogen, C. coccodes, is not yet fully understood (Lees and Hilton 2003). Solanaceous crops such as tomato (Alkan et al. 2012; Lees and Hilton 2003) and weeds from the Cucurbitaceae, Fabaceae, and Solanaceae (Chesters and Hornby 1965) plant families are other known hosts. $C$. coccodes is the causal agent of black dot on potato (Agrios 2005; Johnson and Miliczky 1993). Damage caused by C. coccodes infection of potato was considered to be a minor problem on potato until 1990, when observations of yield losses began (Johnson and Miliczky 1993; Tsror et al. 1999).

Corresponding author: Z. A. Frederick;

E-mail: Zachary.frederick@email.wsu.edu

Accepted for publication 18 February 2017.

() 2017 The American Phytopathological Society
The pale cyst nematode (PCN, Globodera pallida) is also an important potato pathogen (Dandurand 2013; Timmermans et al. 2007). PCN is a regulated pathogen under a Federal Domestic Quarantine Order from USDA Animal and Plant Health Inspection Service and the Idaho State Department of Agriculture (Dandurand 2013, https://www.aphis.usda.gov/aphis/ourfocus/planthealth/plantpest-and-disease-programs/pests-and-diseases/SA_Nematode/sa_ potato/ct_pcn_home). The nematode was identified in southeastern Idaho in 2006 and has become the focus of quarantine and eradication efforts (Dandurand 2013). One possible approach to PCN eradication is the use of trap crops. Trap crops are defined as plants that release root exudates that stimulate nematode egg hatch but are not a host to the nematode (Agrios 2005; Dandurand 2013; Timmermans et al. 2007). Litchi tomato (Solanum sisymbriifolium) is a trap crop for PCN (Dandurand 2013; Scholte and Vos 2000; Timmermans et al. 2007) as well as other nematodes such as Meloidogyne spp. (Dias et al. 2012) and Pratylenchus spp. (Evans and Kerry 2007).

Employing a trap crop such as the litchi tomato as part of PCN eradication may have unintended effects on populations of other soilborne potato pathogens. Litchi tomato was found to be resistant to $V$. dahliae and consequently used as a rootstock for eggplant in Greece (Bletsos et al. 2003). However, the work of Bletsos et al. (2003) should be expanded to include information on the interaction of litchi tomato with $V$. dahliae in other crop production regions, such as North America. It is also important to understand the response of litchi tomato to different host adapted pathotypes of $V$. dahliae compared with that of susceptible potato cultivars in order to determine whether or not the use of litchi tomato as a trap crop for PCN could exacerbate potential Verticillium wilt outbreaks on potato.

The goal of this research was to evaluate the ability of $V$. dahliae host-adapted pathotypes and $C$. coccodes to infect and produce microsclerotia within the stems and roots of litchi tomato, and to quantify microsclerotia production in potato cultivars that are susceptible and moderately resistant to $V$. dahliae relative to litchi tomato. Understanding the potential role of litchi tomato in $V$. dahliae and $C$. coccodes microsclerotia production is important to potato crop rotation sequences if litchi tomato is going to be a PCN trap crop to avoid increasing soilborne potato pathogen inoculum. The objectives of this study were to (i) quantify inoculum production density of two pathotypes of $V$. dahliae and an isolate of $C$. coccodes in stems and roots of greenhouse-grown litchi tomato plants to potato cultivars that differ in susceptibility to $V$. dahliae, and (ii) evaluate litchi 
tomato grown in tandem with potato in field soils infested with endemic $C$. coccodes and $V$. dahliae populations to determine if microsclerotia are formed within stems and roots.

\section{Materials and Methods}

Origin and preparation of isolates. $V$. dahliae and $C$. coccodes isolates were identified and previously characterized for aggressiveness to different hosts and VCG identity by Dung et al. (2013) and Nitzan et al. (2002), respectively (Table 1 ). Inoculum for $V$. dahliae was prepared by placing a single CFU arising from single microsclerotium on semiselective media (NPX) for V. dahliae (Butterfield and DeVay 1977) for 10 days. C. coccodes inoculum was prepared in the same way except that potato dextrose agar (PDA) (Difco Laboratories, Detroit, MI) was used instead of NPX. One subsection of the actively growing border of the colony was excised with a cork borer (7 mm diameter) and one agar disc was placed in Czapek-dox broth (Sigma-Aldrich, St. Louis, MO) for V. dahliae and potato dextrose broth (Difco Laboratories) for $C$. coccodes. Liquid cultures were shaken at $150 \mathrm{RPM}$ at room temperature $\left(21\right.$ to $\left.23^{\circ} \mathrm{C}\right)$ for 10 days. Microsclerotia were generated from this conidial suspension by mixing the suspension with autoclaved sand ( 0.0197 to $0.0234 \mathrm{~mm}$ diameter) and placing the sand/conidia mixture on sterilized mesh in a Pyrex 4,543 ml oblong baking dish (Corning Inc., Corning, NY). The sand/conidia mixture was then placed inside a sterilized enclosure and allowed to grow for 7 days at room temperature (21 to $23^{\circ} \mathrm{C}$ ) before drying for approximately 3 weeks to facilitate the formation of microsclerotia, stirring every $48 \mathrm{~h}$ to prevent clumping.

Determining the minimum inoculum density of $V$. dahliae (CFU/g) in potting medium for litchi tomato infection. The $V$. dahliae inoculum density to be used in subsequent greenhouse inoculations was determined experimentally. The experiment consisted of three plant species (litchi tomato, potato cv. Ranger Russet, and eggplant cv. Night Shadow) by four inoculum levels (5, 30, 50, and $100 \mathrm{CFU} / \mathrm{g}$ ) and one $V$. dahliae isolate (potato pathotype, Table 1) and replicated four times in a completely randomized design. Plants were directly seeded in soilless potting medium (Sunshine Advanced Growing Mix \#4, Sun Gro, Agawam, MA) infested with one isolate of the $V$. dahliae potato pathotype for 4 months before drying for 1 month to allow the formation of microsclerotia. Dried stem sections from the crown to $16 \mathrm{~cm}$ up the plant were collected for $V$. dahliae and $C$. coccodes $\mathrm{CFU}$ enumeration. Roots were not collected in this experiment. Dried stems were ground with an electric coffee grinder (Secura, Appleton, WA) until the particle size was approximately less than $4 \mathrm{~mm}^{2}$. The removable cup from the grinder, as well as the lid, was surface sterilized with a mixture of $70 \%$ ethanol and $30 \%$ deionized water between plant samples. One gram of ground stem was placed evenly on the surface of a Petri plate containing NPX without serial dilution and the number of $V$. dahliae and C. coccodes $\mathrm{CFU}$ was counted under an Olympus SD-ILK dissecting microscope (Olympus Optical Ltd, Tokyo, Japan) after incubating for 10 days.

Quantification of $V$. dahliae and $C$. coccodes CFU in greenhouse grown potato and litchi tomato. Seed of litchi tomato was germinated on moistened filter paper to determine if planted seed was free of $V$. dahliae and $C$. coccodes. Seed free of $V$. dahliae and $C$. coccodes was determined by visually assessing germinated seed for the presence or absence of disease symptoms, sporulation, and microsclerotia associated with either pathogen. Potato tubers from cvs. Alturas, Russet Norkotah, and Ranger Russet were assayed for the absence of both fungal pathogens by slicing an approximately $0.5 \mathrm{~cm}$ thick segment from the stolon end of the tuber, surface sterilized by submerging in $10 \%$ sodium hypochlorite $(\mathrm{NaOCl}, 6.0 \%$, Independent Marketing Alliance, Houston, TX) for $3 \mathrm{~min}$, rinsing in deionized water, and blotting dry. The segment from the stolon end of the tuber was then placed on NPX and incubated at room temperature $\left(21\right.$ to $\left.23^{\circ} \mathrm{C}\right)$ for 10 days, after which the presence or absence of $C$. coccodes and $V$. dahliae microsclerotia was confirmed through visual observation of microsclerotia and sporulation using an Olympus SD-ILK dissecting microscope (Olympus Optical Ltd). Potato tubers that did not test positive for microsclerotial growth and development from either fungal pathogen were planted in 3.79 liter pots with soilless potting medium and allowed to grow for 1 month before the potting medium was infested with $V$. dahliae or $C$. coccodes to ensure emergence. Soils for each litchi tomato and potato plant (experimental unit) were infested with either $V$. dahliae VCG 2B (mint pathotype, Table 1), V. dahliae VCG 4A (potato pathotype, Table 1), or a $C$. coccodes isolate (Table 1 ) so that each pot contained 30 microsclerotia/g potting medium. Litchi tomato and potato seedlings were then directly transplanted into infested potting medium. The greenhouse was maintained at a daytime average of approximately $23^{\circ} \mathrm{C}$ and a nighttime temperature of $15.5^{\circ} \mathrm{C}$.

The experiment had four levels for host (litchi tomato, potato cvs. Russet Norkotah, Alturas, and Ranger Russet) and three levels for pathogen $(V$. dahliae potato pathotype, $V$. dahliae mint pathotype, $C$. coccodes) as well as noninoculated controls arranged in a completely randomized design with four replicates. Cultivar Russet Norkotah is susceptible to V. dahliae (Bae et al. 2007), Ranger Russet is moderately resistant to $V$. dahliae (Bae et al. 2007), and Alturas is resistant to $V$. dahliae in the field (Novy et al. 2003). Soilless potting medium from each experimental unit was infested with one pathogen. Plants were visually inspected for the presence or absence of Verticillium wilt or black dot symptoms once a week for 5 months post inoculation. Plants were harvested in both 2014 and 2015 after 5 months of growth in the greenhouse and slowly dried for 1 month at ambient temperatures of 21 to $24^{\circ} \mathrm{C}$. Slow drying was necessary in order to facilitate the formation of microsclerotia and desiccation of conidia and hyphae. A 16-cm dried stem section from each experimental unit was excised starting from the base of the crown, ground as previously described until particle size was approximately less than $4 \mathrm{~mm}^{2}$, and $1 \mathrm{~g}$ was placed on NPX to enumerate the number of $V$. dahliae or $C$. coccodes $C F U$ that were present after incubation for 10 days as previously described. The greenhouse trial was conducted in the same manner in both 2013 and 2014. V. dahliae or C. coccodes $\mathrm{CFU}$ were enumerated in the stems of all plants in both 2013 and 2014. Root tissue consisting of the combined primary and secondary roots from the crown down $16 \mathrm{~cm}$ was also collected in 2014 for $V$. dahliae or $C$. coccodes CFU enumeration. Root samples were dried, ground, and plated as previously described but separately from stems.

Evaluation of litchi tomato susceptibility to $V$. dahliae and $C$. coccodes under field conditions. Field trials were established to evaluate inoculum production density of $V$. dahliae and $C$. coccodes in infected litchi tomato under field conditions. Research fields were selected based on having a previous history of potato cultivation as well as Verticillium wilt and black dot, with 5 to $15 \mathrm{~V}$. dahliae or C. coccodes microsclerotia per gram of soil based on soil samples. Soil samples were collected from a depth of $10 \mathrm{~cm}$ with a $7 / 8$ " $\times$ 21 " plated soil probe (AMS, American Falls, ID), dried for 1 month

Table 1. Verticillium dahliae and Colletotrichum coccodes isolates used to confirm host status of litchi tomato (Solanum sisymbriifolium) for either V. dahliae or C. coccodes

\begin{tabular}{lccccc}
\hline Pathogen & Host-adapted pathotype & State of origin & Original plant host & VCGy $^{\mathbf{y}}$ & Source citation \\
\hline V. dahliae & Potato & Idaho & Potato seed tuber & $4 \mathrm{~A}$ & Dung et al. 2013 \\
V. dahliae & Mint & Washington & Peppermint & 2B & Dung et al. 2013 \\
C. coccodes & $-\mathrm{z}$ & Montana & Potato seed tuber & 3 & Nitzan et al. 2002 \\
\hline
\end{tabular}

\footnotetext{
${ }^{\mathrm{y}}$ Vegetative compatibility group.
}

${ }^{\mathrm{z}}$ Not considered for this study. 
at ambient temperatures of 21 to $24^{\circ} \mathrm{C}$, and $1 \mathrm{~g}$ of dry soil was placed on NPX, incubated for 10 days, and $V$. dahliae or $C$. coccodes microsclerotia were quantified as previously described for dry plant matter.

An observational study to evaluate inoculum production density of $V$. dahliae and C. coccodes in infected litchi tomato under field conditions was established in Othello, WA, in 2014 and Powell Butte, $\mathrm{OR}$, in 2015. Natural levels of inoculum ( $>5 \mathrm{~V}$. dahliae or C. coccodes microsclerotia) already present in the soil were used in both field trials. A one-row plot was established in Othello, WA, with five replicates containing four potato plants (cv. Ranger Russet) and three litchi tomato plants. The row was $9 \mathrm{~m}$ long row with $0.9 \mathrm{~m}$ alleys, with plant spacing of $25 \mathrm{~cm}$ for both potato and litchi tomato. Potato seed tubers were planted on 28 April and litchi tomato was planted on 28 May 2014 in Othello, WA. Flowers of litchi tomato were removed in July and August to prevent seed dispersal. In September 2014, all plants in Othello, WA, were individually harvested and dried slowly for 3 weeks in the dark at temperatures of 21 to $24^{\circ} \mathrm{C}$ to facilitate the formation of microsclerotia. Thirty litchi tomato plants were planted on 15 May 2015 in Powell Butte, OR, approximately $45 \mathrm{~cm}$ apart. Plants were harvested after 4 months in the field on 23 September, dried as previously described. Stem and root sections $16 \mathrm{~cm}$ in length were excised from dried potato or litchi tomato and ground as in previously described greenhouse experiments. Dried plant matter was placed evenly on the surface of NPX to determine the number of $V$. dahliae and C. coccodes CFU from the stem and roots of each experimental unit after 10 days' incubation as previously described.

Field trials with potato cv. Russet Burbank and litchi tomato were established in Prosser, WA, in 2015 to evaluate and compare inoculum production density of $V$. dahliae and $C$. coccodes in infected litchi tomato to potato under field conditions. Russet Burbank and litchi tomato were planted in the field at Prosser, WA, in a randomized complete block design. This experiment consisted of six litchi tomato or six potato plants per replicate with six replicates for a total of 72 experimental units. Potato and litchi tomato transplants approximately 15 to $18 \mathrm{~cm}$ tall were planted approximately $25 \mathrm{~cm}$ apart in June and harvested in September 2015. Stem and root sections $16 \mathrm{~cm}$ in length were excised from dried potato or litchi tomato and ground as previously described for the greenhouse experiments. Dried plant matter was placed evenly on the surface of NPX to determine the number of $V$. dahliae and $C$. coccodes $\mathrm{CFU}$ from the stem and roots of each experimental unit after 10 days' incubation as previously described.

Data analysis. Analysis of variance (ANOVA) was performed in SAS University Edition (SAS Inc., Cary, NC) followed by

Table 2. Verticillium dahliae potato pathotype microsclerotia counts (CFU/g) from eggplant, potato, or litchi tomato (Solanum sisymbriifolium) stems at senescence by initial $V$. dahliae colony forming units (CFU)/g potting medium

\begin{tabular}{lcccc}
\hline & \multicolumn{4}{c}{ V. dahliae CFU/g potting medium } \\
\cline { 2 - 5 } & $\mathbf{5}$ & $\mathbf{3 0}$ & $\mathbf{5 0}$ & $\mathbf{1 0 0}$ \\
\hline Potato cv. Ranger Russet & $49.5 \mathrm{bc}^{\mathrm{z}}$ & $55.6 \mathrm{ab}$ & $72.9 \mathrm{ab}$ & $114.5 \mathrm{a}$ \\
Eggplant cv. Night Shadow & $6.4 \mathrm{c}$ & $27.0 \mathrm{bc}$ & $16.6 \mathrm{bc}$ & $39.9 \mathrm{bc}$ \\
Litchi tomato & $2.7 \mathrm{c}$ & $4.1 \mathrm{c}$ & $8.1 \mathrm{c}$ & $10.6 \mathrm{bc}$ \\
\hline
\end{tabular}

${ }^{\mathrm{z}}$ Letters denote mean separation by Tukey's honestly significant difference test for all pairwise comparisons across columns and rows $(P \leq 0.05)$.
Tukey's honestly significant difference test for all pairwise comparisons to determine differences in the number of CFU observed between plants subjected to the same fungal pathogen, separated by plant part (stem or root). Greenhouse trial data were not pooled prior to analysis. Fields from Prosser, WA, were pooled prior to analysis (with ANOVA), with each field serving as the replicates. $V$. dahliae and $C$. coccodes data were analyzed separately because the experiment was designed to compare inoculum production density of a single pathogen between the four plant hosts. ANOVA assumptions were verified using the same program. Data sets that did not initially meet ANOVA assumptions were log transformed to allow continued use of parametric analysis, except for values of zero.

\section{Results}

Determining the minimum inoculum density of $V$. dahliae (CFU/g) in potting soil for litchi tomato infection. The number of $V$. dahliae CFU in litchi tomato and eggplant did not differ regardless of the inoculum level in the potting medium $(5 \mathrm{CFU} / \mathrm{g}[P=$ 0.9972], $30 \mathrm{CFU} / \mathrm{g}[P=0.84], 50 \mathrm{CFU} / \mathrm{g}[P=0.9967], 100 \mathrm{CFU} / \mathrm{g}$ $[P=0.53]$, Table 2). Eggplant was not used to compare differences in $V$. dahliae inoculum production density because of nonsignificant differences in the number of observed $V$. dahliae CFU between eggplant and litchi tomato at any evaluated inoculum level (Table 2). $V$. dahliae infection of litchi tomato was observed at all levels of $V$. dahliae inoculum but litchi tomato had significantly fewer observed $V$. dahliae CFU than potato cv. Ranger Russet at inoculation levels of $30 \mathrm{CFU} / \mathrm{g}(P<0.0001), 50 \mathrm{CFU} / \mathrm{g}(P<0.0001)$, and $100 \mathrm{CFU} / \mathrm{g}$ $(P<0.0001)$ (Table 2). Therefore, in subsequent experiments, plants were inoculated with $30 \mathrm{CFU} / \mathrm{g}$, which was the minimum $V$. dahliae inoculum density that consistently infected litchi tomato and had significantly fewer observed CFU than potato.

Quantification of $V$. dahliae and $C$. coccodes $\mathrm{CFU}$ in greenhouse grown potato and litchi tomato. The two-factor ANOVA test for differences in the number of microsclerotia between plant hosts was significant $(P<0.0001)$, and the interaction effect of plant host and fungal isolate was not significant $(P=0.3115)$. Greater numbers of CFUs were recorded for the $V$. dahliae potato pathotype than the mint pathotype on cv. Ranger Russet $(P=0.0331)$, Alturas $(P<0.0001)$, and Russet Norkotah $(P<0.0001)$ in 2013 (Table 3). Cultivars Alturas $(P=0.0003)$, Ranger Russet $(P=0.0193)$, and Russet Norkotah $(P=$ 0.0022) produced greater numbers of $V$. dahliae potato pathotype CFUs than litchi tomato (Table 3). Litchi tomato planted in soilless mix infested with either pathotype of $V$. dahliae was infected, based on the production of microsclerotia within the plants, but no difference in CFUs was observed between the potato and mint pathotypes of $V$. dahliae $(P=0.1044$, Table 3$)$. No Verticillium wilt symptoms were observed on litchi tomato inoculated with either the $V$. dahliae mint or potato pathotype (data not shown) in 2013.

The two-factor ANOVA test for differences in the number of microsclerotia between plant hosts was significant $(P<0.0001$ for both stems and roots), and the interaction effect of plant host and fungal isolate was not significant $(P=0.1058$ in stems, $P=0.27$ for roots). Greater numbers of $\mathrm{CFU}$ of the $V$. dahliae potato pathotype were observed than the mint pathotype in cvs. Russet Norkotah $(P=$ $0.0432)$ and Ranger Russet $(P=0.0029)$ roots in 2014 (Table 4). Significantly greater numbers of $V$. dahliae potato pathotype CFU were found in stems and roots of cv. Russet Norkotah than litchi tomato

Table 3. Mean number of Verticillium dahliae microsclerotia from stems of three potato cultivars Alturas, Russet Norkotah, and Ranger Russet, and litchi tomato in a greenhouse experiment in 2013

\begin{tabular}{lcccc}
\hline & \multicolumn{3}{c}{ Mean CFU/g stem $^{\mathbf{z}}$} \\
\cline { 2 - 5 } Isolate & Alturas & Russet Norkotah & Ranger Russet & Litchi tomato \\
\hline Potato pathotype & $97.0 \mathrm{a}$ & $107.8 \mathrm{a}$ & $72.0 \mathrm{a}$ & \\
Mint pathotype & $46.3 \mathrm{~b}$ & $20.5 \mathrm{c}$ & $4.5 \mathrm{~d}$ & \\
Noninoculated control & $5.0 \mathrm{~d}$ & $18.5 \mathrm{c}$ & $0.2 \mathrm{~d}$ & $0.8 \mathrm{~d}$ \\
\hline
\end{tabular}

${ }^{\mathrm{z}}$ Letters denote mean separation by ANOVA followed by Tukey's honestly significant difference test for all pairwise comparisons for $V$. dahliae CFU counts across columns and rows $(P \leq 0.05)$. 
$(P=0.0001$, Table 4$)$. This is in contrast to litchi tomato having fewer $V$. dahliae CFU of either pathotype than all potato cultivars in 2013 (Table 3). The number of $V$. dahliae potato and mint pathotype CFUs did not differ in litchi tomato stems $(P=0.3962)$ or roots $(P=1.0)$ in 2014 (Table 4). No Verticillium wilt symptoms were observed on litchi tomato inoculated with either the $V$. dahliae mint or potato pathotype (data not shown) in 2014.

The number of observed CFUs of $C$. coccodes from stems was significantly lower in litchi tomato than potato cv. Alturas $(P=0.0057)$, Russet Norkotah $(P=0.0041)$, and Ranger Russet $(P=0.0041)$ in 2013 (Table 5). No differences were noted between the $C$. coccodes CFUs from stems or roots of any of the potato cvs. as well as litchi tomato roots compared with potato roots in $2014(P>0.05$, Table 5). No black dot symptoms were observed on litchi tomato inoculated in the greenhouse experiments (data not shown) in either 2013 or 2014.

Evaluation of litchi tomato susceptibility to $V$. dahliae and $C$. coccodes under field conditions. Greater numbers of CFUs of $V$. dahliae were observed in stems $(P=0.0307)$ and roots $(P=$ 0.0465) of cv. Russet Burbank than the litchi tomato in the Prosser, WA, field trial (Table 6). The numbers of CFUs of $C$. coccodes did not differ in stems $(P=0.6296)$ and roots $(P=0.0576)$ of cv. Russet Burbank when compared with litchi tomato at this site (Table 6). Both pathogens infected and produced microsclerotia in litchi tomato in the Othello, WA, field trial in 2014 and Powell Butte, OR, field trial in 2015 (Table 6). Comparisons could not be made between C. coccodes or $V$. dahliae CFU between potato or litchi tomato because of insufficient field replication in Othello, WA, and no potato plants were planted in Powell Butte, OR. No symptoms of either pathogen were observed on litchi tomato at harvest in the field (data not shown).

\section{Discussion}

Litchi tomato was confirmed as a host for $V$. dahliae and C. coccodes as indicated by the isolation of $V$. dahliae and $C$. coccodes from the stems and roots of plants grown in both greenhouse and field settings. Inoculum production density of the $V$. dahliae potato and mint pathotypes from litchi tomato did not significantly differ, which indicates the potato pathotype was not consistently more aggressive on litchi tomato. The difference in aggressiveness between the $V$. dahliae potato and mint pathotypes was replicated on the potato cultivars in the greenhouse as evidenced by the increased numbers of observed CFU produced on the stems or roots of the Verticilliumsusceptible cv. Russet Norkotah. In 2014, Russet Norkotah seed pieces were unexpectedly infected with $V$. dahliae and produced microsclerotia; however, further improvements and diligence in checking seed pieces for infection reduced the number of microsclerotia observed in the subsequent trials. Because plant samples were dried before grinding, the CFU observed on the semiselective media (NPX) were likely derived from $V$. dahliae or $C$. coccodes microsclerotia instead of conidia or hyphae.

The potato cv. Russet Norkotah is highly susceptible to $V$. dahliae (Bae et al. 2007), which explains why this cultivar consistently had the greatest numbers of $V$. dahliae $\mathrm{CFU} / \mathrm{g}$ tissue. Observations of decreased inoculum production densities in litchi tomato stems in 2013 and roots in 2014 compared with cv. Russet Norkotah led to the initial conclusion that litchi tomato was less susceptible to $V$. dahliae than a susceptible potato cultivar. However, litchi tomato did not consistently have significantly greater $V$. dahliae inoculum production density than $\mathrm{cv}$. Ranger Russet, a cultivar that is moderately resistant to $V$. dahliae infection (Bae et al. 2007). The lack of a difference in inoculum production densities of $V$. dahliae potato pathotype between Ranger Russet and litchi tomato in 2014 was probably due to partial resistance on the part of both Ranger Russet and litchi tomato.

The litchi tomato plants used in this experiment were not expected to be genetically uniform because the seeds used to produce them were from selected intermated plants. The difference in $V$. dahliae resistance in litchi tomato likely varied between years due to the genetic

Table 6. Mean number of Verticillium dahliae or Colletotrichum coccodes CFU from stems of potato and litchi tomato (Solanum sisymbriifolium) in the 2014 field trial in Othello, WA, and the 2015 field trials in Prosser, WA, and Powell Butte, OR

\begin{tabular}{|c|c|c|c|c|c|c|}
\hline \multirow[b]{2}{*}{ Year } & \multirow[b]{2}{*}{ Location } & \multirow[b]{2}{*}{ Plant } & \multicolumn{2}{|c|}{ V. dahliae } & \multicolumn{2}{|c|}{ C. coccodes } \\
\hline & & & Stem $^{w}$ & $\operatorname{Root}^{w}$ & Stem $^{w}$ & $\operatorname{Root}^{n}$ \\
\hline \multirow[t]{2}{*}{2015} & Prosser, WA & Potato $^{x}$ & $26.6 \mathrm{a}$ & $49.6 \mathrm{a}$ & $7.6 \mathrm{a}$ & $19.6 \mathrm{a}$ \\
\hline & Prosser, WA & Litchi tomato & $5.4 \mathrm{~b}$ & $18.9 \mathrm{~b}$ & $2.3 \mathrm{a}$ & $1.6 \mathrm{a}$ \\
\hline \multirow[t]{2}{*}{2014} & Othello, WA & Potato $^{z}$ & 22.4 & 16.8 & 18.9 & 23.3 \\
\hline & Othello, WA ${ }^{\mathrm{y}}$ & Litchi tomato & 1 & 1.1 & 0 & 0 \\
\hline 2015 & Powell Butte, OR & Litchi tomato & 20 & 3.3 & 43.3 & 30 \\
\hline
\end{tabular}

${ }^{\mathrm{w}}$ Letters denote mean separation by ANOVA followed by a Tukey's honestly significant difference test for all pairwise comparisons within columns only $(P \leq 0.05)$. Each pathogen and each plant part were analyzed separately.

$x$ Potato cv. Russet Burbank.

y No statistical test conducted (insufficient replication).

z Potato cv. Ranger Russet.

Table 4. Mean number of Verticillium dahliae CFU from stems and roots of three potato cultivars Alturas, Russet Norkotah Ranger Russet, and litchi tomato (Solanum sisymbrifolium) in a greenhouse in 2014

\begin{tabular}{|c|c|c|c|c|c|}
\hline \multirow[b]{2}{*}{ Plant part } & \multirow[b]{2}{*}{ V. dahliae isolate } & \multicolumn{4}{|c|}{ Mean CFU/g plant part $\mathrm{z}$} \\
\hline & & Alturas & Russet Norkotah & Ranger Russet & Litchi tomato \\
\hline \multirow[t]{3}{*}{ Stem } & Potato pathotype & $40.8 \mathrm{abc}$ & $88.4 \mathrm{a}$ & $25.4 \mathrm{abcd}$ & $6.1 \mathrm{cdef}$ \\
\hline & Mint pathotype & $22.5 \mathrm{abcd}$ & 19.5 abcde & $6.2 \mathrm{def}$ & 12.4 ef \\
\hline & Noninoculated control & $2.3 \mathrm{f}$ & $2.0 \mathrm{f}$ & $0.6 \mathrm{f}$ & $1.1 \mathrm{f}$ \\
\hline \multirow[t]{3}{*}{ Root } & Potato pathotype & $57.8 \mathrm{ab}$ & $87.4 \mathrm{a}$ & $30.4 \mathrm{ab}$ & $20.5 \mathrm{~b}$ \\
\hline & Mint pathotype & $31.7 \mathrm{ab}$ & $17.8 \mathrm{bc}$ & $3.6 \mathrm{~cd}$ & $22.0 \mathrm{~b}$ \\
\hline & Noninoculated control & $2.3 \mathrm{~cd}$ & $0.3 \mathrm{~d}$ & $1.4 \mathrm{~cd}$ & $1.9 \mathrm{~cd}$ \\
\hline
\end{tabular}

${ }^{\mathrm{z}}$ Letters denote mean separation by ANOVA followed by a Tukey's honestly significant difference test for all pairwise comparisons for $V$. dahliae CFU counts across columns and rows $(P \leq 0.05)$.

Table 5. Mean number of Colletotrichum coccodes CFU from stems and roots of three potato cultivars Alturas, Russet Norkotah Ranger Russet, and litchi tomato (Solanum sisymbriifolium) in a greenhouse in 2013 and 2014

\begin{tabular}{|c|c|c|c|c|c|c|}
\hline \multirow[b]{2}{*}{ Year } & \multirow[b]{2}{*}{ Plant part } & \multirow[b]{2}{*}{ Pathogen } & \multicolumn{4}{|c|}{ Mean CFU/g plant part ${ }^{z}$} \\
\hline & & & Alturas & Russet Norkotah & Ranger Russet & Litchi tomato \\
\hline 2013 & Stem & C. coccodes & $40.5 \mathrm{a}$ & $19.8 \mathrm{~b}$ & $19.8 \mathrm{~b}$ & $3.0 \mathrm{c}$ \\
\hline \multirow{2}{*}{2014} & Stem & C. coccodes & $56.0 \mathrm{a}$ & $77.0 \mathrm{a}$ & $28.8 \mathrm{a}$ & $14.8 \mathrm{a}$ \\
\hline & Root & C. coccodes & $56.0 \mathrm{a}$ & $79.3 \mathrm{a}$ & $33.0 \mathrm{a}$ & $46.1 \mathrm{a}$ \\
\hline
\end{tabular}

${ }^{\mathrm{z}}$ Letters denote mean separation by ANOVA followed by a Tukey's honestly significant difference test for all pairwise comparisons for $C$. coccodes $\mathrm{CFU}$ counts across the 2013 row and for all columns and rows in $2014(P \leq 0.05)$. Each year was analyzed separately. 
variability in seed. Different sets of litchi tomato plants that were evaluated in 2013 and 2014 varied in $V$. dahliae resistance, and these plants also varied in resistance to different pathotypes of $V$. dahliae. For example, comparisons of the mint pathotype of $V$. dahliae on roots in 2014 revealed that lower inoculum production density within Ranger Russet than litchi tomato but the same trend was not observed when inoculum was the mint pathotype and litchi tomato plants used in 2013.

The inconsistencies in litchi tomato resistance to the two specific $V$. dahliae pathotypes leaves no clear trend that has emerged across both years. Resistance on the part of litchi tomato could be imparted on certain seedlings, and not others, by genetic variability in resistance genes. The case can be made for partial resistance since litchi tomato plants were infected but resulted in quantitatively less inoculum production than susceptible potato cultivars. Additionally, symptoms of black dot did not develop on inoculated litchi tomato plants. Litchi tomato could be a source of resistance that would be useful in breeding programs for solanaceous crops where Verticillium wilt is a limiting factor to potato production. Only populations of litchi tomato selected for resistance to $V$. dahliae should be used as a trap crop for nematodes.

Greater C. coccodes inoculum production was observed in potato cvs. Alturas, Russet Norkotah, and Ranger Russet than litchi tomato in 2013. However, no differences between any of the three potato cultivars and litchi tomato were observed in 2014 due to high variability in the number of microsclerotia observed in all plant hosts between replicates. The inconsistency in less $C$. coccodes inoculum production density in litchi tomato stems compared with Alturas and Russet Norkotah is a possible indicator of partial resistance in some individual litchi tomato plants to $C$. coccodes as plants were infected in 2013 but with quantitatively less inoculum than susceptible potato. As with litchi tomato resistance to $V$. dahliae, possible litchi tomato resistance to $C$. coccodes could be imparted on certain seedlings, and not others, by genetic variability in resistance genes.

Litchi tomato was infected with $V$. dahliae in all three field sites in Othello and Prosser, WA, and Powell Butte, OR. In field trials in Prosser, WA, litchi tomato produced fewer $V$. dahliae CFUs as a result of natural infection than potato cv. Ranger Russet. Litchi tomato infection with $C$. coccodes was observed in Prosser, WA, and Powell Butte, OR, but not Othello, WA, which could support litchi tomato was partially resistant to infection by $C$. coccodes or a disease escape occurred. However, litchi tomato planted at the Prosser, WA, site did not produce fewer $C$. coccodes $\mathrm{CFU}$ s as a result of natural infection than potato cv. Russet Burbank, suggesting not all litchi tomato plants are resistant to infection by $C$. coccodes. Variation in severity of infection by $C$. coccodes and $V$. dahliae between field sites is likely due to differences in inoculum density, but could also be influenced by weather and irrigation events. Consistent trends in susceptibility to either pathogen between field sites is important for identifying resistance in litchi tomato. Numerically, greater numbers of $V$. dahliae and $C$. coccodes inoculum production densities were sometimes observed in litchi tomato grown in the greenhouse than in the field, and perhaps this is due to greater levels of inoculum (30 CFU/g) or an environment more conducive for infection by one or both pathogens in the greenhouse.

Litchi tomato was confirmed as a host for V. dahliae and C. coccodes, and the variation in inoculum production densities between the potato and mint pathotypes of $V$. dahliae indicate that neither pathotype is consistently more aggressive on litchi tomato. Observations of decreased $V$. dahliae inoculum densities in litchi tomato compared with potato cv. Russet Norkotah but not Ranger Russet suggests that litchi tomato was partially resistant to $V$. dahliae like Ranger Russet. The inconsistency in decreased $C$. coccodes inoculum production density in litchi tomato stems compared with potato cvs. Alturas and Russet Norkotah is evidence supporting some induvial plants of litchi tomato could also be partially resistant to $C$. coccodes. Variation in resistance within the litchi tomato population is expected given that the litchi tomato seeds were from an intermated selected population that was genetically variable. Further supporting evidence of litchi tomato partial resistance to both pathogens is the lack of visible disease symptoms for either pathogen in greenhouse trials when plants were artificially inoculated.

The use of litchi tomato as a PCN trap crop is likely to have limited effect on the proliferation of $V$. dahliae or $C$. coccodes populations in the soil. Slattery (1981) documented an increase of microsclerotia in soil over several seasons in the Red River Valley of Minnesota and North Dakota with the use of the potato cv. Kennebec that returned relatively high numbers of microsclerotia to the soil. The conclusions from Slattery (1981) indicate that the amount of $V$. dahliae inoculum produced on preceding crops and potato cultivars has influence on the severity of Verticillium wilt in following potato crops (Slattery 1981). Care would certainly be needed to manage both $V$. dahlaie and $C$. coccodes in a field planting of litchi tomato since litchi tomato can be infected by both pathogens and possibly return microsclerotia inoculum to the soil, albeit less than a susceptible potato cultivar such as Russet Norkotah. Only populations of litchi tomato selected for resistance to $V$. dahliae and $C$. coccodes should be used as a trap crop for nematodes.

\section{Acknowledgments}

This work was supported, in part, by funds appropriated to Washington State University, Pullman, WA, by the Washington State Potato Commission. PPNS \# 0712, Department of Plant Pathology, College of Agricultural, Human, and Natural Resource Sciences, Agricultural Research Center, Hatch Project No. WNP0678, Washington State University, Pullman, WA 99164-6430, U.S.A. We would like to thank Dr. Inga Zasada for planting litchi tomato in Powell Butte, OR. We appreciate David Wheeler, Dr. Debra Inglis, and Dr. Weidong Chen for editorial suggestions before submission.

\section{Literature Cited}

Agrios, G. N. 2005. Plant Pathology, 5th Ed. Elsevier Academic Press, San Diego, CA.

Alkan, N., Fluhr, R., and Prusky, D. 2012. Ammonium secretion during Colletotrichum coccodes infection modulates salicylic and jasmonic acid pathways of ripe and unripe tomato fruit. Mol. Plant-Microbe Interact. 25:85-96.

Bae, J., Atallah, Z. K., Jansky, S. H., Rouse, D. I., and Stevenson, W. R. 2007. Colonization dynamics and spatial progression of Verticillium dahliae in individual stems of two potato cultivars with differing responses to potato early dying. Plant Dis. 91:1137-1141.

Bhat, R. G., Smith, R. F., Koike, S. T., Wu, B. M., and Subbarao, K. V. 2003. Characterization of Verticillium dahliae isolates and wilt epidemics of pepper. Plant Dis. 87:789-797.

Bhat, R. G., and Subbarao, K. V. 1999. Host range specificity in Verticillium dahliae. Phytopathology 89:1218-1225.

Bletsos, F., Thanassoulopoulos, C., and Roupakias, D. 2003. Effect of grafting on growth, yield, and Verticillium wilt of eggplant. HortScience 38:183-186.

Butterfield, E. J., and DeVay, J. E. 1977. Reassessment of soft assays for Verticillium dahliae. Phytopathology 67:1073-1078.

Chesters, C. G. C., and Hornby, D. 1965. Studies on Colletotrichum coccodes 2. Alternative host tests and tomato fruit inoculations using a typical tomato root isolate. Trans. Br. Mycol. Soc. 48:583-594.

Dandurand, L. M. 2013. Novel eradication strategies for pale cyst nematode. Potato Progress vol. 13, no 10. Online: https://idahopotato.com/uploads/ media/documents/2013-09-16-potato-progress.pdf

Dias, M. C., Conceição, I. L., Abrantes, I., and Cunha, M. J. 2012. Solanum sisymbriifolium - a new approach for the management of plant-parasitic nematodes. Eur. J. Plant Pathol. 133:171-179.

Dung, J. K., Peever, T. L., and Johnson, D. A. 2013. Verticillium dahliae populations from mint and potato are genetically divergent with predominant haplotypes. Phytopathology 103:445-459.

Evans, K., and Kerry, B. 2007. Changing priorities in the management of potato cyst nematodes. Outlooks Pest. Manage. 18:265-269.

Fordyce, C., and Green, K. J. 1960. Studies of host specificity of Verticillium alboatrum var. menthae. Phytopathology 50:635.

Joaquim, T. R., and Rowe, R. C. 1990. Reassessment of vegetative compatibility relationships among strains of Verticillium dahliae using nitrate-nonutilizing mutants. Phytopathology 80:1160-1166.

Joaquim, T. R., and Rowe, R. C. 1991. Vegetative compatibility and virulence of strains of Verticillium dahliae from soil and potato plants. Phytopathology 81: 552-558.

Johnson, D. A., and Miliczky, E. R. 1993. Effects of wounding and wetting duration on infection of potato foliage by Colletotrichum coccodes. Plant Dis. 77:13-17.

Katan, T. 2000. Vegetative compatibility in populations of Verticillium-an overview. Pages 69-86 in: Advances in Verticillium: Research and Disease Management. E. C. Tjamos, R. C. Rowe, J. B. Heale, and D. R. Fravel, eds. APS Press, St. Paul, MN 
Lees, A. K., and Hilton, A. J. 2003. Black dot (Colletotrichum coccodes): an increasingly important disease of potato. Plant Pathol. 52:3-12.

Nitzan, N., Hazanovsky, M., Tal, M., and Tsror, L. 2002. Vegetative compatibility groups in Colletotrichum coccodes, the causal agent of black dot on potato. Phytopathology 92:827-832.

Novy, R. G., Corsini, D. L., Love, S. L., Pavek, J. J., Mosley, A. R., James, S. R., and Thornton, R. E. 2003. Alturas: a multi-purpose, russet potato cultivar with high yield and tuber specific gravity. Am. J. Potato Res. 80: 295-301.

Pegg, G. F., and Brady, B. L., eds. 2002. Verticillium wilts. CABI Bioscience Publishing, New York. doi:10.1079/9780851995298.0000
Scholte, K., and Vos, J. 2000. Effects of potential trap crops and planting date on soil infestation with potato cyst nematodes and root-knot nematodes. Ann. Appl. Biol. 137:153-164

Slattery, R. J. 1981. Inoculum potential of Verticillium-infested potato cultivars. Am. J. Potato Res. 58:135-142.

Timmermans, B. G. H., Vos, J., Van Nieuwburg, J., Stomph, T. J., Van der Putten, P. E. L., and Molendijk, P. G. 2007. Field performance of Solanum sisymbriifolium, a trap crop for potato cyst nematodes. Ann. Appl. Biol. 150:89-97.

Tsror, L., Erlich, O., and Hazanovsky, M. 1999. Effect of Colletotrichum coccodes on potato yield, tuber quality, and stem colonization during spring and autumn. Plant Dis. 83:561-565. 